\title{
EFFECTS OF POLYASPARTIC ACID ON REMINERALIZATION OF INTRAFIBRILLAR DENTIN AND SIZE OF HYDROXYAPATITE CRYSTALS
}

\author{
WAVIYATUL AHDI ${ }^{1}$, NILAKESUMA DJAUHARIE ${ }^{2 *}$, RATNA MEIDYAWATI ${ }^{2}$, IKHWAN MUSLIM ${ }^{1}$ \\ ${ }^{1}$ Conservative Dentistry Residency Program, Faculty of Dentistry, Universitas Indonesia, Indonesia. ${ }^{2}$ Department of Conservative \\ Dentistry, Faculty of Dentistry, Universitas Indonesia, Indonesia. Email: nila.setyopurnomo@gmail.com
}

Received: 09 October 2019, Revised and Accepted: 05 July 2020

\section{ABSTRACT}

Objective: The objective of this study was to analyze the intrafibrillar remineralization process and the size of hydroxyapatite crystals formed.

Methods: The samples were divided into four groups. The control group was immersed in a demineralization solution, whereas the remaining three treatment groups were immersed in a remineralization solution containing polyaspartic acid for 3,7 , or 14 days. The effect of polyaspartic acid on intrafibrillar remineralization during the polymer-induced liquid-precursor (PILP) process was evaluated using transmission electron microscopy, and the size of hydroxyapatite crystals was examined through X-ray diffraction.

Results: Significant differences were detected in the intrafibrillar remineralization between the treatment groups (subjected to remineralization for 3,7 , and 14 days) and the demineralized dentin control group. However, no significant differences were detected in the size of hydroxyapatite crystals between the control and the treatment groups.

Conclusion: Our data suggest that polyaspartic acid enables intrafibrillar remineralization during the PILP process.

Keywords: Dentin remineralization, Polyaspartic acid, Non-collagenous protein.

(C) 2020 The Authors. Published by Innovare Academic Sciences Pvt Ltd. This is an open access article under the CC BY license (http://creativecommons. org/licenses/by/4. 0/) DOI: http://dx.doi.org/10.22159/ijap.2020.v12s2.OP-24

\section{INTRODUCTION}

Dentin caries is classified into infected dentin and affected dentin $[1,2]$. Infected dentin is the outermost layer of caries consisting of a mineral matrix and hyrdroxyapatite crystals which are destroyed by a bacterial infection and its byproducts. The damage is irreversible and inhibits remineralization. By contrast, affected dentine is the inner layer that is partially demineralized, but the damage is still reversible. The affected dentine zone can undergo demineralization as it contains healthy collagen fibrils surrounded by hydroxyapatite crystals and collagen crosslinks [2,3]. Dentin remineralization can occur due to the deposition of minerals between collagen fibers [4].

Remineralization can occur through two methods: Conventional remineralization and guided-tissue remineralization (GTR). Conventional remineralization occurs through epitaxial growth of mineral crystals present in demineralized lesions [5], whereas GTR is a method of extrafibrillar and intrafibrillar collagen dentin mineralization in calcium phosphate phase [6]. Non-collagen proteins play a very important role in stabilizing the amorphous calcium phosphate (ACP) formation which does not form aggregates and stays in nano [4,7]. Dentin matrix protein 1 (DMP1), a noncollagen protein with a high affinity for calcium ions, is actively involved in regulating mineral formation by interacting with collagen fibrils through electrostatic bonds and plays a key role in mineral formation in the intrafibrillar and extrafibrillar spaces [8]. However, DMP1 can be damaged by caries, and an analogous material is needed to replace the damaged DMP1. Analogous materials for remineralization must contain multiple phosphate and carboxyl groups that can bind to calcium [7]. Several such analogous materials are known to be able to mimic the function of DMP1 non-collagen protein [4].

Recently, polymer-induced liquid-precursor (PILP) has been developed as a process involved in GTR $[2,9,10]$. This process is known as a rapid biomimetic remineralization process and uses synthetic anionic polymer materials that can replace the role of non-collagen proteins in intrafibrillar remineralization process $[11,12]$. These polymers play an important role in the phase of liquid mineral precursors. Fluids easily fill the gaps in collagen fibrils, with the remineralization of collagen fibrils, and the resulting nanodroplets (15-30 nm diameter) diffusing into type 1 collagen intrafibrillar or gap zone $[2,13]$. Polyaspartic acid, a non-collagen protein analog necessary for the PILP process, is a negatively charged amino acid synthesized using non-toxic and biodegradable materials $[4,14]$.

Remineralization of intrafibrillar collagen can be visualized using transmission electron microscopy (TEM). A TEM is an electron microscope that works by passing electrons through the object of observation and obtains information in the form of images [7,15]. In addition, the average size and crystallinity of a sample can be examined using the X-ray diffraction (XRD) technique [5].

\section{METHODS}

The research protocol used in this study (Protocol No. 0512111218) was approved by the Ethics Committee of Faculty of Dentistry, Universitas Indonesia. After extraction, the teeth were immediately immersed in deionized water at a temperature of $40^{\circ} \mathrm{C}$. The samples were divided into four groups. Group I (control) represented demineralized dentin (without remineralization), whereas Groups II, III, and IV (treatment groups) were subjected to remineralization for 3,7 , and 14 days, respectively.

In the control group, the dentin block was soaked in demineralization solution ( $40 \mathrm{ml}$ of $0.05 \mathrm{M}$ acetate buffer containing $2.2 \mathrm{mM}$ calcium phosphate [pH 5]) for $66 \mathrm{~h}$. In the treatment groups, $40 \mathrm{ml}$ of remineralization solution containing $50 \mathrm{mM}$ of Tris buffer, $0.9 \% \mathrm{NaCl}$, $0.02 \% \mathrm{NaN}_{3}, 4.5 \mathrm{mM} \mathrm{CaCl} 2$, and $2.1 \mathrm{mM} \mathrm{K}_{2} \mathrm{HPO}_{4}$ was used. Then, $4 \mathrm{mg}$ of polyaspartic acid powder (molecular weight $=23 \mathrm{kDa}, 100 \mu \mathrm{g} / \mathrm{ml}$; 
Alamanda Polymer USA) was mixed into the solution and incubated at $37^{\circ} \mathrm{C}$ by shaking it continuously for 3,7 , or 14 days. The samples were analyzed using XRD to determine the size of hydroxyapatite crystals and TEM to observe intrafibrillar remineralization, which can be detected based on the occurrence of mineral deposits in dentin collagen intrafibrillar.

The data were analyzed using the normality distribution test in each group using the Shapiro-Wilk test. Furthermore, the Kruskal-Wallis test was used to determine differences among the four groups. All statistical analyses were carried out using the SPSS 22 software, at a significance level of $\mathrm{p}<0.05$.

\section{RESULTS AND DISCUSSION}

In this study, the remineralization of demineralized dentin using polyaspartic acid in the PILP process was evaluated using XRD and TEM. The TEM was performed to visualize the occurrence of intrafibrillar remineralization characterized by mineral deposits in the intrafibrillar collagen. The XRD examination was carried out to evaluate the size of hydroxyapatite crystals [16].

In all groups, the size of hydroxyapatite crystals was smaller than $40 \mathrm{~nm}$ (Table 1), i.e., smaller than the size of the gap zone filled with hydroxyapatite crystals in collagen fibrils, as shown previously [1,7]. However, the number of hydroxyapatite crystals is more important than the size. Crystals nucleate and grow to a certain extent depending on the Gibbs free energy [5]. The relatively small size of crystals $(<40 \mathrm{~nm})$ in all samples indicates that remineralization has entered the intrafibrillar area to produce nanometer crystals. Next, we performed TEM to visualize the occurrence of intrafibrillar remineralization.

Fig. 1 shows an elongated amorphous demineralized dentine, which indicates the presence of collagen (blue arrow) and a few crystals, which appeared as black dots (black arrows). Demineralization that occurs is partially demineralized. In general, crystals in intrafibrillar collagen still remain [1].

Table 1: Average size of hydroxyapatite crystals in various groups

\begin{tabular}{lllll}
\hline Sample & \multicolumn{4}{l}{ Hydroxyapatite crystal size (nm) } \\
\cline { 2 - 5 } & $\begin{array}{l}\text { Demineralized } \\
\text { dentin control group }\end{array}$ & \multicolumn{3}{l}{ Remineralization groups } \\
\cline { 3 - 5 } & & $\mathbf{3}$ days & $\mathbf{7}$ days & $\mathbf{1 4}$ days \\
\hline Sample 1 & 30.455 & 30.987 & 25.375 & 38.381 \\
Sample 2 & 19.449 & 29.933 & 8.180 & 25.379 \\
Sample 3 & 30.455 & 25.997 & 25.384 & 30.458 \\
Sample 4 & 27.109 & 32.659 & 25.376 & 30.457 \\
\hline
\end{tabular}

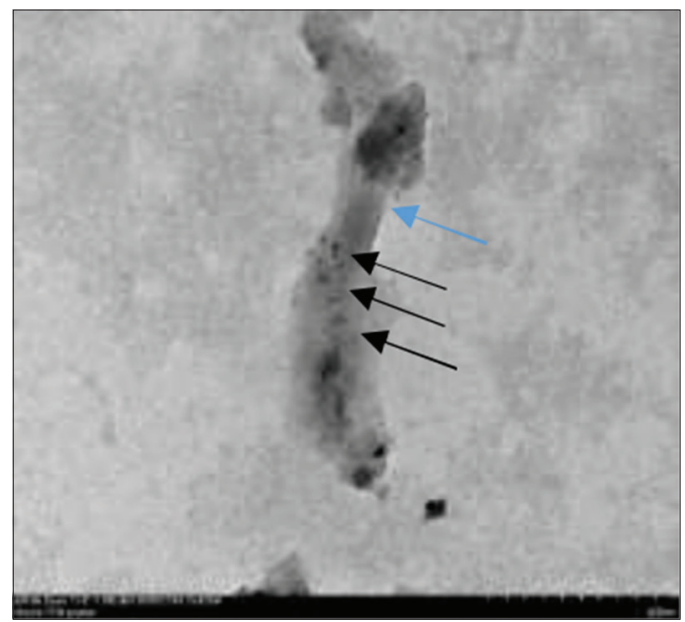

Fig. 1: Transmission electron microscopy of demineralized dentine
Fig. 2 shows regular elongated collagen (blue arrow) and the presence of more black dots (black arrows) indicating the entry of mineral deposits in the intrafibrillar space. This proves that intrafibrillar remineralization has begun.

Collagen observed after 7 days of remineralization was elongated and striated (Fig. 3, blue arrow) resembling collagen in healthy dentine. In addition, several black dots were observed in the collagen indicating an increase in the number of mineral deposits that entered the gap zone (black arrows).

Increasingly dark collagen was observed after 14 days of remineralization (Fig. 4, blue arrow) indicating that mineral deposits that entered the gap zone filled the collagen intrafibrillar space (black arrows). Changes in the TEM images (Figs. 1-4) indicate remineralization of intrafibrillar collagen.

In this study, we aimed to determine the occurrence of dentine remineralization after the application of polyaspartic acid, which was able to remineralize the affected dentine during the PILP process and resulted in intrafibrillar and extrafibrillar remineralization $[13,17]$.

Remineralization of dentine using the GTR method involves reconstruction of two components: Type 1 collagen and apatite minerals. Collagen functions as a scaffold for the deposition of apatite [4,7]. In addition, non-collagen proteins play an important role in binding to collagen and stabilizing ACP by preventing the

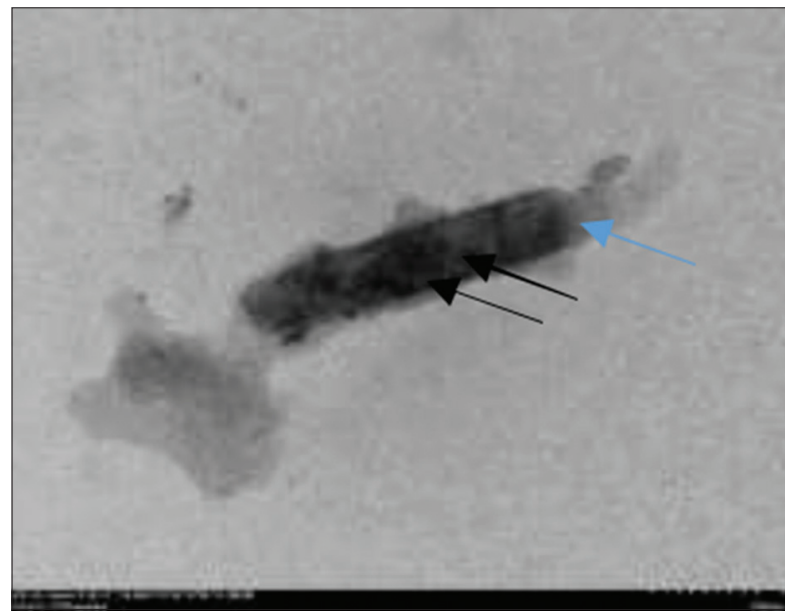

Fig. 2: Transmission electron microscopy of Group II (remineralization for 3 days)

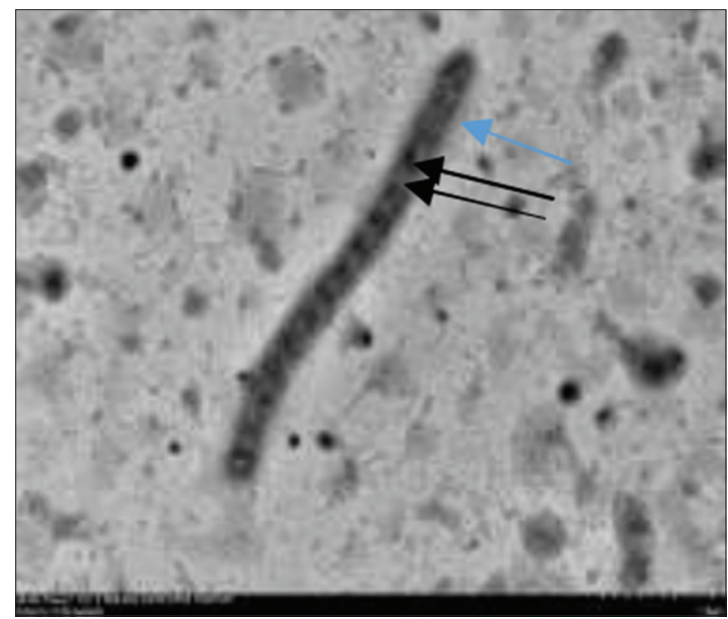

Fig. 3: Transmission electron microscopy of Group III (remineralization for 7 days) 


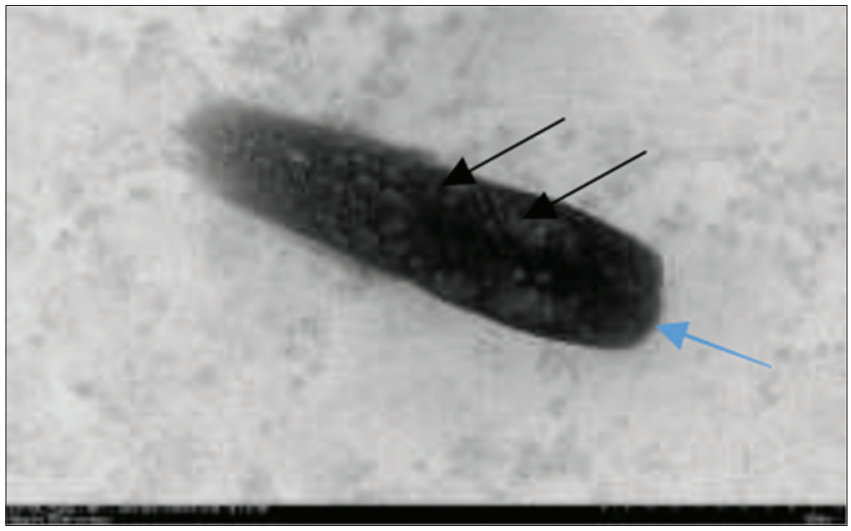

Fig. 4: Transmission electron microscopy of Group IV (remineralization for 14 days)

formation of aggregates. Then, the nanocomplex penetrates the gaps between collagen fibers and forms hydroxyapatite crystals resulting in intrafibrillar remineralization $[6,7]$.

In this study, polyaspartic acid was used as a non-collagen protein analog, as it is biocompatible, biodegradable, and non-toxic. Because of the presence of a carboxyl group, polyaspartic acid showed a high affinity for calcium ions $[4,6]$. Polyaspartic acid also plays a role in stabilizing the ACP by preventing its aggregation and maintaining its nanosize. Then, the nanocomplex entered intrafibrillar collagen and forms hydroxyapatite crystals resulting in intrafibrillar remineralization [5]. According to the previous studies, excess intrafibrillar remineralization improves the mechanical properties of dentine [1,2]. Intrafibrillar minerals are more resistant to demineralization, and dentin collagen with high intrafibrillar mineral concentration has a high modulus of elasticity [4].

The size of hydroxyapatite crystals in each sample was smaller than $40 \mathrm{~nm}$, as examined by XRD (Table 1) [18] . Fibrils contain a 40-nm gap zone, which is filled with intrafibrillar minerals [18]. The size of hydroxyapatite crystals below $40 \mathrm{~nm}$ implies that the crystals can enter the gap zone or collagen intrafibrillar space [1]. No significant differences were detected in the size of hydroxyapatite crystals among the four groups; this is consistent with the theory of Gibbs free energy, which states that crystals have a limited ability to grow [15]. The size below $40 \mathrm{~nm}$ also proves that polyaspartic acid, the non-collagen protein analog used in this study, can maintain ACP formation and prevents the aggregation of ACP nanoparticles into larger particles [6]. The polyaspartic acid-ACP nanocomplex is capable of penetrating the gap zone and forming hydroxyapatite crystals.

To ensure the occurrence of intrafibrillar remineralization, we performed TEM [7]. The use of polyaspartic acid as a substitute for DMP1 to induce intrafibrillar remineralization is evident by TEM (Figs. 1-4). Fig. 1 shows irregular collagen, indicating that collagen is degraded by the loss of most minerals. The demineralization is partial; therefore, some crystals remain in the intrafibrillar collagen. Collagen acts as a scaffold for the nucleation and growth of hydroxyapatite crystals [7]. Fig. 2 shows the entry of calcium and phosphate mineral deposits in the intrafibrillar space after 3 days of remineralization indicating the start of intrafibrillar remineralization. Mineral precursors could enter into the gap zone and diffuse along fibrils within $24 \mathrm{~h}$. Furthermore, apatite crystals are formed along collagen fibrils on horse tendons within $72 \mathrm{~h}$ [13]. The collagen in Fig. 3 resembled that in healthy dentine with elongated and striated features after 7 days of remineralization. In addition, many black dots appeared in the collagen, indicating an increase in the number of mineral deposits in the gap zone. This indicates that the remineralization of intrafibrillar collagen increases with time. Fig. 4 shows the presence of darker collagen after 14 days of remineralization indicating that the mineral deposits that enter the gap zone gradually fill the intrafibrillar collagen space.

The formation of ACP precursors is an important stage in biomineralization [2]. Fibril collagen acts as a scaffold for intrafibrillar mineralization $[1,4,19]$. Gower et al. (2012) showed that ACP diffuses into the collagen in the liquid form via capillary action [13]. However, infiltration of ACP into collagen is mediated by the interaction of the charge between the complex minerals and certain areas of collagen fibrils $[4,13]$. Interaction occurs between the negatively charged polymer-mineral complex and positively charged gap zone [5]. ACP precursors stabilized by the negatively charged polymers in the form of nanodroplets interact with positive charges along with collagen molecules and stimulate compaction and nucleation of ACP in collagen. The nucleation of ACP develops and matures into apatite nanocrystals along with the collagen intrafibrillar space $[4,19]$. This was clearly observed by TEM, especially in Fig. 4.

PILP is known as a fast remineralization process and uses synthetic anionic polymers that can replace the role of non-collagen proteins in the intrafibrillar remineralization process $[2,20]$. Polyaspartic acid plays an important role in remineralization because it is able to bind to positively charged calcium ions through its negatively charged carboxyl group $[4,6]$. Our PILP study provides a strong foundation for developing dental materials that play a key role in the remineralization of dentine intrafibrillar collagen; for example, a superior restoration material for the remineralization of intrafibrillar dentine collagen in the caries affected by dentine. Further research is needed to investigate the strength of collagen formed by remineralization.

\section{CONCLUSION}

The size of hydroxyapatite crystals formed after 3,7 , and 14 days of remineralization was similar in size (average $20-30 \mathrm{~nm}$ ). Intrafibrillar remineralization started on the $3^{\text {rd }}$ day and increased with time, as indicated by the presence of mineral deposits on intrafibrillar collagen after remineralization with polyaspartic acid in the PILP process.

\section{REFERENCES}

1. Uskoković V, Bertassoni LE. Nanotechnology in dental science: Moving towards a finer way of doing dentistry. Materials 2010; 3:1674-91.

2. Nurrohman H, Carneiro KM, Hellgeth J, Saeki K, Marshall SJ, Marshall GW, et al. The role of protease inhibitors on the remineralization of demineralized dentin using the PILP method. PLoS One 2017;12:1-15.

3. Mazzoni A, Tjaderhane L, Checchi V, Lenarda RD, Salo T, Pashley DH, et al. Role of dentin MMPs in caries. J Dent Res 2015;94:241-51.

4. Niu LN, Zhang W, Pashley DH, Breschi L, Mao J, Chen JH, et al. Biomimetic remineralization of dentin. Dent Mater 2014;30:77-96.

5. Zhang X, Neoh KG, Lin CC, Kishen A. Remineralization of partially demineralized dentine substrate based on a biomimetic strategy. J Mater Sci Mater Med 2012;23:733-42.

6. Chen Z, Cao S, Wang H, Li Y, Kishen A, Deng X. Biomimetic remineralization of demineralized dentine using scaffold of CMC/ACP nanocomplexes in an in vitro tooth model of deep caries. PLos One 2015;10:e0116553.

7. Bertasoni LE, Habelitz S, Kinney JH, Marshall SJ, Marshall GW Jr. Biomechanical perspective on the remineralization of dentin. Caries Res 2009;43:70-7.

8. Manuel T, Estrella O, Inmaculada C, Raquel O. Early dentine remineralization: Morpho-mechanical assessment. J Dent 2014;42:384-94.

9. Thalia T, Felicia S, Douglas R, Laurie G. Mimicking the nanostructure of bone: Comparison of polymeric process-directing agents. Polymers 2011;3:10-35

10. Bedran-Russo AK, Ravindran S, George A. Imaging analysis of early DMP1 mediated dentine remineralization. Arch Oral Biol 2013;58:254-60.

11. Zargar V, Asghari M, Dashti A. A review on chitin and chitosan polymers : Structure, chemistry, solubility, derivatives, and applications. ChemBioEng Rev 2015;2:124.

12. Bedran-Russo AK, Pauli GF, Chen SN, McAlpine J, Castellan CS. 
Dentin biomodification: Strategies, renewable resources and clinical application. Dent Mater 2014;30:62-76.

13. Gower LB, Burwell AK, Thula-Mata T. Functional remineralization of dentin lesions using polymer-induced liquid-precursor process. PLoS One 2012;7:e38852.

14. Osorio R, Osorio E, Cabello I, Toledano M. Zinc induces apatite and scholzite formation during dentin remineralization. Caries Res 2014;48:276-90.

15. Li R, Zhang X, Dong H, Li Q, Shuai Z, Hu W. Gibbs-curie-wulff theorem in organic materials: A case study on the relationship between surface energy and crystal growth. Adv Mater 2015;28:1697-702.
16. Bunaciu AA, Udriștioiu EG, Aboul-Enein HY. X-ray diffraction: Instrumentation and applications. Crit Rev Anal Chem 2015;45:289-99.

17. Nurrahman H, Saeki K, Karneiro KM, Chien YC. Repair of dentin from DSPP KO mice by PILP mineralization. J Mater Res 2016;31:321-7.

18. Ingham B. X-ray scattering characterisation of nanoparticles. Crystallogr Rev 2015;21:229-303.

19. Zhao J, Liu Y, Sun W, Zhang H. Amorphous calcium phosphate and its application in dentistry. Chem Cent J 2011;5:40.

20. Krogstad D, Wang D, Gibson SL. Kinetics of aggregation and crystallization of polyaspartic acid stabilized calcium phosphate particles at high concentrations. Biomacromolecules 2015;16:1550-5. 